\title{
PET-CT VISUALIZATION OF INTRACRANIAL LIPOMAS
}

\author{
Yavor Enchev', Ara Kaprelyan ${ }^{2}$, Pavel Bochev ${ }^{3}$, Elitsa Encheva ${ }^{4}$, Borislav Chaushev ${ }^{3}$, \\ Bogomil Iliev ${ }^{1}$, Alexandra Tzoukeva ${ }^{2}$ \\ ${ }^{1}$ Department of Neurosurgery, Medical University of Varna \\ ${ }^{2}$ Department of Neurology, Medical University of Varna \\ ${ }^{3}$ Department of Nuclear Medicine, Medical University of Varna \\ ${ }^{4}$ Department of Imaging Diagnostics and Radiotherapy, Medical University of Varna
}

\begin{abstract}
Intracranial lipomas are rare lesions, infrequently indicated for neurosurgical treatment due to their benign course and significant surgery-related complications rate. The aim of this study was to collect, describe and analyze the patients with intracranial lipomas verified by PET-CT and reported in the literature up to date. The literature search was performed through Pub Med using the combination of the terms intracranial lipomas, CT, MRI and PET-CT. The thorough review of the relevant papers did not find even one case with PET-CT images of intracranial lipomas. The first two personal illustrative cases were demonstrated by our report. The value of PET-CT in the primary and differential diagnosis of intracranial lipomas was analyzed, in comparison with CT, MRI and PET-MRI.
\end{abstract}

Keywords: CT, intracranial lipoma, MRI, PET-CT, PET-MRI

\section{INTRODUCTION}

Intracranial lipomas are infrequent lesions, occasionally indicated for neurosurgical treatment due to their benign course and high surgery-related morbidity. Thus, the precise preoperative identification of these lesions is with apparent practical importance. The noninvasive imaging diagnosis of intracranial lipomas is mainly based on CT and MRI

Address for correspondence:

Yavor Enchev, MD, PhD

Clinic of Neurosurgery

University Hospital "St. Marina",

1 "Hristo Smirnenski" Blvd,

9010 Varna, Bulgaria

phone: +359-888 441191

e-mail:dr.y.enchev@gmail.com

Received: March 03, 2015

Accepted: June 10, 2015 studies. The presented personal cases are the first reported in the literature with PET-CT images of intracranial lipomas, even obtained unintentionally. Due to the continuously growing number of patients that underwent cranial PET-CT for other reasons, the radiologist, neurologist, oncologist and neurosurgeons must be familiar with the typical PET-CT features of intracranial lipomas.

\section{CASE REPORTS}

Case 1.

A 32-year-old male with complex partial and secondary generalized epileptic seizures from childhood was admitted to our hospital. Unenhanced CT revealed two lesions- a pericallosal mass $38 / 50 \mathrm{~mm}$ in size (Figure 1, A1) and a periinsular tumor with dimensions of $10 / 6 \mathrm{~mm}$ (Figure 1, A2), both with homogenous low attenuation values and perilesional calcifications. These findings led to a diagnosis of 
intracranial lipomas. Subsequent MRI and PET-CT were performed for complete diagnostic clarification of epileptic seizures (Figure 1).

Case 2.

A 72-year-old male with tremor of the left extremities was referred to our clinic. Unenhanced CT exposed pericallosal lesion with dimensions $41 / 10 \mathrm{~mm}$ and features of intracranial lipoma (Figure 1, B). Consequent MRI and PET-CT were done for further etiologic diagnosis of the tremor.

In both cases, software-based PET/MRI fusion was performed intentionally for this study.

\section{DISCUSSION}

\section{Literature review}

The study represented a short case report and thorough clinical review of the scant literature pertinent to the imaging of intracranial lipomas. The literature search was performed through Pub Med using the combination of the terms intracranial lipomas, CT, MRI and PET-CT. The relevant papers were reviewed in order to obtain data for the target cases (1-8).

However, the literature review revealed no cases of PET-CT imaging, except the presented personal illustrative cases which are outlined in Table 1.
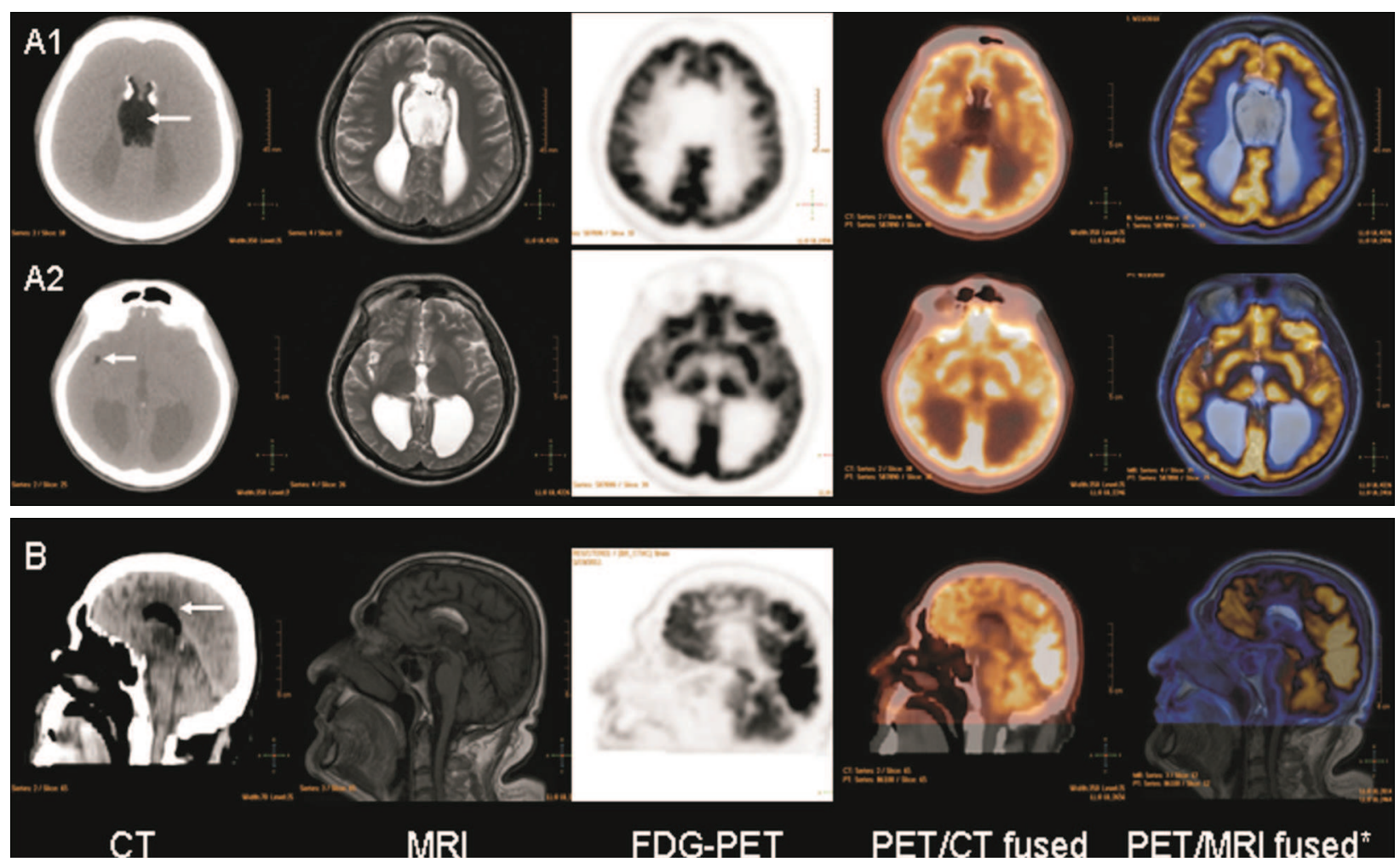

Figure 1 A, B: (A1) A 32-year-old male (Case 1). Previous CT revealed lipomas of the brain, confirmed by MRI. FDG PET/CT reveals a central interhemispheric mass with fat-equivalent density and peripheral calcifications. The lesion is severely hypometabolic, with an activity lower than that of white matter. Surrounding cingulate cortex has a normal metabolic pattern. (A2) A second lipoma is detected close to right insula. The lesion is indistinguishable on metabolic images alone. However direct fusion with CT and MRI shows the region is hypometabolic. (B) A 72-years-old male (Case 2). Previous CT revealed lipoma of the brain, confirmed by MRI. FDG PET/CT reveals central interhemispheric mass with fat-equivalent density. The mass is severely hypometabolic, with an activity lower than that of white matter. ${ }^{*}$ Fused PET/MRI image contains PET and MRI images, acquired from different machines, registered by interactive software algorithm on Phillips Fusion viewer 
Intracranial lipomas most probably represent congenital malformations caused by anomalous differentiation of persistent primitive meninges and comprise up to $1 \%$ of all intracranial tumors (1-3). Histologically intracranial lipomas are vastly vascularized, outlined by collagenous capsule, with or without calcifications, which may be thoroughly adherent to the neighboring neurovascular structures and contain centrally located adult fat cells with peripheral, occasionally indented nuclei. Most frequently intracranial lipomas are localized in the pericallosal region followed by the quadrigeminal, suprasellar, interpeduncular, cerebellopontine and sylvian cisterns (1). Seldom intracranial lipomas may be associated with other intracranial (mainly callosal agenesis or hypoplasia) and/or extracranial developmental abnormalities. They are benign, mainly asymptomatic lesions which may infrequently lead to headache, epilepsy, cranial nerve palsy and behavioral disorders. The diagnosis of intracranial lipomas is primarily based on CT and MR imaging (4-7). However, up to date, to the best of our knowledge, PET$\mathrm{CT}$ images of intracranial lipomas have never been reported in the literature.

Preoperative diagnosis of intracranial lipomas

Surgical removal of intracranial lipomas generally is not recommended because of the significant complications rate and benign course of these lesions. Thus, the preoperative diagnosis of intracranial lipomas is of utmost importance (1-3).

\section{CT diagnosis of intracranial lipomas}

Intracranial lipomas have a distinctive appearance on unenhanced CT $(4,5)$, with low attenuation values ranging between -40 Hounsfield Units (HU) and $-80 \mathrm{HU}$ (mean - $60 \mathrm{HU}$ ). Calcifications are often present in interhemispheric lipomas, most commonly in their fibrous capsule.

\section{MR diagnosis of intracranial lipomas}

Intracranial lipomas have a characteristic high signal on T1-weighted images and intermediate to low signal on T2-weighted spin-echo sequences on Magnetic Resonance Imaging (MRI) $(2,5,8)$. The differential diagnosis includes epidermoid and dermoid cysts (7) 
Yavor Enchev, Ara Kaprelyan, Pavel Bochev et al.

\section{PET-CT diagnosis of intracranial lipomas}

Nowadays, CT and MRI are widely accepted cross-sectional imaging techniques for the initial diagnosis and follow-up of patients with intracranial lipomas. An additional imaging technique in the evaluation of these patients could be PET as molecular imaging using radiopharmaceuticals that are incorporated into metabolic pathways of normal and abnormal cells.

In contrast to CT and MRI tumor diagnosis, which is dependent on changes in morphology (shape, size), electron density (CT), or proton density (MRI) in abnormal tissue, 18FFDG (Fluorine18fluorodeoxyglucose) PET tumor detection is based on changes in glucose metabolism in tumor cells (8). Logically, abnormal metabolic changes at a cellular level (PET) should be detectable before macroscopic morphologic changes (CT and/or MRI). However, PET is limited by poor spatial resolution that may make it difficult to accurately localize 18FFDG uptake to an anatomic structure. This limitation has been significantly reduced by the introduction of combined PET-CT scanner. Addition of CT to PET has shown better specificity and sensitivity than either PET or CT alone as it has the ability to demonstrate functional and structural details in the same setting (8).

The presented cases are the first reported in the literature PET-CT images of intracranial lipomas. PET-CT was not performed intentionally to diagnose the intracranial lipomas, but on other occasion. The well-known drawbacks of the PET-CT are the low image resolution, the low spatial resolution, the radiation burden, the high price, the limited availability, the big study duration. The CT and MRI definitely outrivaled PET-CT in the diagnosis of intracranial lipomas.

PET-MRI diagnosis of intracranial lipomas or future perspectives

PET/MRI is a hybrid imaging technology that incorporates MRI soft tissue morphological imaging and PET functional imaging. At the moment less than 20 facilities all over the world have installed this technology. Some of the main clinical fields of PET/ MRI are neurology and neurosurgery. Nowadays, the research studies are actively conducted to evaluate the expected advantages of the new PET/MRI diagnostic method compared to PET-CT. Thus, the presented software-based PET/MRI fused images of intracranial lipomas are also unique in the literature.

\section{Drawbacks of the current study}

The number of the patients in the current series unfortunately is quite limited and obviously insufficient for drawing valid conclusions on the analyzed problem.

\section{CONCLUSION}

In summary, although PET-CT is never going to be a gold standard in imaging diagnosis of intracranial lipomas, the familiarity of the subject could be with practical value. In the absence of preceding CT and/or MRI images, PET-CT could be a reliable tool in the primary or differential diagnosis of these lesions. Indisputably, nowadays PET-CT does not have the capacity to become the imaging of choice for intracranial lipomas, due to its genuine drawbacks.

\section{REFERENCES}

1. Yildiz H, Hakyemez B, Koroglu M, Yesildag A, Baykal B. Intracranial lipomas: importance of localization. Neuroradiology. 2006;48:1-7.

2. Loddenkemper T, Morris III HH, Diehl B. Intracranial lipomas and epilepsy. J Neurol. 2006;253:590-593.

3. Yilmaz N, Unal O, Kiymaz N, Yilmaz C, Etlik O. Intracranial lipomas- a clinical study. Clinical Neurol Neurosurg. 2006;108:363-368.

4. Hayashi T, Shojima K, Moritaka K. Intracranial lipomas- case presentations and CT features. $\mathrm{Ku}$ rume Med J. 1983;30:133-141.

5. Ichikava T, Kumazaki T, Mizumura S, Kijima T, Motohashi S, Gocho G. Intracranial lipomasdemonstration by computed tomography and magnetic resonance imaging. J Nihon Med Sch. 2000;67:388-391.

6. Truwit CL, Barkovich AJ. Pathogenesis of intracranial lipoma: an MR study in 42 patients. AJR Am J Roentgenol. 1990;155:855-864.

7. Warakaulle DR, Anslow P. Differential diagnosis of intracranial lesions with high signal on T1 or low signal on T2 weighted MRI. Clinical Radiology. 2003; 58:922-933.

8. Kapoor V, McCook BM, Torok FS. An introduction to PET-CT imaging. RadioGraphics. 2004;24:523-543. 